\title{
SOME WEAKLY INNER AUTOMORPHISMS OF THE CUNTZ ALGEBRAS
}

\author{
SZE-KAI TSUI
}

(Communicated by Palle E. T. Jorgensen)

\begin{abstract}
Let $V$ be an $n \times n$ unitary matrix. Then $V$ induces an automorphism $O_{V}$ of $O_{\infty}$ and $O_{n}$. It is shown in this paper that $O_{V}$ is weakly inner. Let $F_{n}$ be the UHF $C^{*}$-subalgebra of $O_{n}$ and $U$ be a unitary operator in the diagonal maximal abelian ${ }^{*}$-subalgebra of $F_{n}$. Then the automorphism $\lambda_{U}$ of $O_{n}$ defined by $\lambda_{U}\left(S_{i}\right)=U^{*} S_{i}$, for each generator $S_{i}$ of $O_{n}$, is weakly inner. Any automorphism conjugate to either one mentioned above is also weakly inner.
\end{abstract}

\section{INTRODUCTION}

In 1977, J. Cuntz studied a class of separable simple infinite $C^{*}$-algebras [3], which are denoted by $O_{n}$ 's for $n=2,3, \ldots O_{n}$ is also a crossed product of a UHF-subalgebra $F_{n}$ (of $O_{n}$ ) by a single endomorphism of $F_{n}$, scaling the trace of $F_{n}$. Since 1977 there has been a good amount of work on various properties of $O_{n}$. In this paper we concentrate on some automorphisms of $O_{n}$ and $O_{\infty}$ (see the definition in Section 2). Earlier works exhibited some outer automorphisms [1], [2], [6], [7]. A theorem proved by Kishimoto [10] in answering a longstanding question raised in [11] by $\mathrm{C}$. Lance states that an automorphism of a separable simple unital $C^{*}$-algebra is inner if and only if it is universally weakly inner (see the definition in Section 2). Here we try to determine which outer automorphisms are weakly inner (see the definition in Section 2) and succeed in describing a large class of weakly inner automorphisms, containing outer automorphisms studied in [2], [6], [7]. The notion of weak innerness can first be found in [9]. The results are contained in Sections 3 and 4.

\section{NotATION AND SOME LEMMAS}

Let $H$ be an infinite-dimensional Hilbert space. For $n=2,3, \ldots$, let $\left\{S_{1}, \ldots, S_{n}\right\}$ be $n$ isometries on $H$ such that

$$
\sum_{i=1}^{n} S_{i} S_{i}^{*}=I .
$$

Received by the editors July 19, 1993 and, in revised form, September 13, 1993.

1991 Mathematics Subject Classification. Primary 46L40, 46L60.

Key words and phrases. Cuntz algebras, weakly inner automorphisms, FOCK representation, separable simple $C^{*}$ algebras, universally weakly inner automorphisms, outer automorphisms, permutations. 
Let $O_{n}$ be the $C^{*}$-algebra generated by $S_{1}, \ldots, S_{n}$. For $n=\infty$ let $\left\{S_{i}\right\}_{i=1}^{n}$ be a sequence of isometries on $H$ such that

$$
\sum_{i=1}^{k} S_{i} S_{i}^{*} \leq I \quad \text { for } k=1,2, \ldots
$$

Let $O_{\infty}$ be the $C^{*}$-algebra generated by $\left\{S_{i}\right\}_{i=1}^{\infty}$. It turns out that the definition of $O_{n}$ for $n=1,2, \ldots, \infty$ is space free and depends only on a set of isometries satisfying (2.1) or (2.2) (see 1.12 in [3]). These algebras are contained in every simple $C^{*}$-algebra which contains an infinite projection [4]. Following the notation by Cuntz in [3], for $k=0,1, \ldots$ we let $W_{k}^{n}$ be the set of all $k$-tuples $\left(j_{1}, \ldots, j_{k}\right)$ with $j_{i} \in\{1, \ldots, n\}$, for $i=1, \ldots, n$, and $W=\bigcup_{k=0}^{\infty} W_{k}^{n}$ with $W_{0}^{n}=\{0\}$. We write $S_{0}=1$, and for $\mu=\left(j_{1}, \ldots, j_{k}\right) \in W_{k}^{n}$ we set $S_{\mu}=S_{j_{1}} \cdots S_{j_{k}}$ and $l(\mu)=k$.

For a finite positive integer $n \geq 2, O_{n}$ has a $C^{*}$-subalgebra, $F_{n}$, the closed linear span of terms of the form $S_{\mu} S_{\nu}^{*}$ with $l(\mu)=l(\nu)$, which is a UHF-algebra of type $n^{\infty}$. Consider

$$
F_{n}=\bigcup_{k=1}^{\infty} \mathbb{A}_{k},
$$

where

$$
\mathbb{A}_{k}=\bigotimes_{i=1}^{k} M_{n_{i}}
$$

and $n_{i}=n$ for $i=1, \ldots, k$, and $M_{n}$ is the algebra of $n \times n$ (complex) matrices. Let $D_{k}$ be the subalgebra of all diagonal matrices of $\mathbb{A}_{k}$ and

$$
D=\bigcup_{k=1}^{\infty} D_{k} .
$$

$D$ is a maximal abelian subalgebra in both $F_{n}$ and $O_{n}$, and $D$ is also the closed linear span of terms of the form $S_{\mu} S_{\mu}^{*}$ for $\mu \in W_{k}^{n}$, for $k=0,1, \ldots$. Let $P$ be the conditional expectation of $O_{n}$ onto $D$. In the following definition we write $S$ for $S_{1}$ and $S^{-1}$ for $S_{1}^{*}$. Let

$$
\mathbb{A}=\left\{\sum_{i=-n}^{-1} S^{i} A_{i}+A_{0}+\sum_{i=1}^{n} A_{i} S^{i} \mid A_{i} \in \bigcup_{k=1}^{\infty} \mathbb{A}_{k}\right\} .
$$

It is easy to see that $\mathbb{A}$ is a norm-dense ${ }^{*}$-algebra of $O_{n}$. Let $\alpha$ be any endomorphism of $O_{n}$ such that $\alpha(1)=1$. Then there is a unitary element $Z_{\alpha} \in O_{n}$ such that $\alpha\left(S_{i}\right)=Z_{\alpha}^{*} S_{i} \quad(i=1,2, \ldots, n)$. In fact, $Z_{\alpha}^{*}=\sum_{i=1}^{n} \alpha\left(S_{i}\right) S_{i}^{*}$. On the other hand, every unitary element $U \in O_{n}$ determines a unique isometric endomorphism $\lambda_{U}$ of $O_{n}$ such that $\lambda_{U}\left(S_{i}\right)=U^{*} S_{i} \quad(i=1, \ldots, n)$ and $\lambda_{U}(1)=1$. This was first observed by $M$. Takesaki and communicated to J. Cuntz. From Proposition 1.1 in [5] we know that $Z_{\alpha}$ satisfies a cocycle identity, $\lambda$ is a twisted representation, and $\lambda_{U}$ is an automorphism if and only if there is a unitary $V \in O_{n}$ such that $\lambda_{U}(V)=U^{*}$. Let $\Phi$ denote the endomorphism of $O_{n}$ defined by $\Phi(x)=\sum_{i=1}^{n} S_{i} x S_{i}^{*}$. Let $V=\left(V_{i j}\right)$ be a unitary operator on $C_{n}$, the $n$-dimensional complex Hilbert space. Then it is easy to check that $\left\{T_{i}=\sum_{k=1}^{n} V_{k i} S_{k} ; i=1,2, \ldots, n\right\}$ generate the same $C^{*}$-algebra $O_{n}$ and $V$ 
induces on $O_{n}$ an automorphism $O_{V}$ by $O_{V}\left(S_{i}\right)=T_{i}$, for $i=1,2, \ldots, n$. In 1979, Archbold studied the case $n=2$ and $V=\left(\begin{array}{l}01 \\ 10\end{array}\right)$ and found $O_{V}$ is outer yet weakly inner [1]. An automorphism $\beta$ of a $C^{*}$-algebra $A$ is said to be universally weakly inner if $\beta^{* *}$ on $A^{* *}$ is inner, and $\beta$ is said to be weakly inner if there exists a faithful representation $\pi$ of $A$ such that, in identifying $A$ and $\pi(A), \beta$ extends to an inner automorphism on the weak-operator closure $\overline{\pi(A)}^{w}$. Subsequently it was shown in [6], [7] that $O_{V}$ is outer for all unitary $V$ in $C^{n}$ for $n=2,3, \ldots, \infty$. In 1981, Kishimoto [10] resolved a longstanding question with an affirmative answer, that is, an automorphism on a separable simple unital $C^{*}$-algebras is inner if and only if it is universally weakly inner. Thus the remaining question for $O_{V}$ for $n=3, \ldots, \infty$ and unitary $V$ is whether $O_{V}$ 's are weakly inner. One consequence of the results in this paper is that $O_{V}$ 's are all weakly inner.

First we show a lemma which will lead us to a desired representation, $\pi$, of $O_{n}$ in which a given automorphism has an inner extension to the weak-operator closure of $\pi\left(O_{n}\right)$.

2.3. Lemma. Let $A$ be a simple $C^{*}$-algebra and $\beta$ be an automorphism of $A$. Suppose that $\varphi$ is a $\beta$-invariant state of $A$, i.e., $(\varphi \circ \beta)(x)=\varphi(x)$ for all $x$ in $A$. Then $\beta$, the automorphism of the image $\pi_{\varphi}(A)$ of $A$ under the GNS representation $\pi_{\varphi}$ induced by $\varphi$, can be extended to an automorphism $\hat{\beta}$ on the weak-operator closure of $\pi_{\varphi}(A)$. Furthermore $\hat{\beta}$ is spatial.

Proof. Let $H_{\varphi}$ be the GNS representation space of $\pi_{\varphi}$ and $f$ be the cyclic vector of $\pi_{\varphi}(A)$ with $\varphi(x)=\left\langle\pi_{\varphi}(x) f, f\right\rangle$ for all $x$ in $A$. The operator $U$ defined on $H_{\varphi}$ by $U\left(\pi_{\varphi}(x) f\right)=\pi_{\varphi}(\beta(x)) f(x \in A)$ is a unitary operator. Let $\hat{\beta}(x)=U x U^{*}$ for $x \in \bar{\pi}_{\varphi}(A)$. It is easy to see that $\left.\hat{\beta}\right|_{\pi_{\varphi}(A)}=\beta$. Q.E.D.

2.4. Remark. Let $\alpha, \beta$ be two automorphism of a simple $C^{*}$-algebra $A$. If a pure state $\varphi$ is $\beta$-invariant, then $\varphi_{0} \alpha$ is $\alpha^{-1} \beta \alpha$-invariant and pure. It is now clear that to establish weak innerness of a given automorphism $\beta$ of $A$ it is enough to find a pure state $\varphi$ and an automorphism $\alpha$ such that $\varphi$ is $\alpha^{-1} \beta \alpha$-invariant.

\section{SOME WEAKLY INNER AUTOMORPHISMS ON $O_{n}, n$ : FINITE}

Let us first observe that $\Phi\left(\mathbb{A}_{k}\right) \subseteq \mathbb{A}_{k+1}$ for all $k=1,2, \ldots$, and $\Phi(x) S_{i}=$ $S_{i} x$ for all $x$ in $O_{n}, i=1, \ldots, n$.

3.1. Lemma. Let $U$ be a unitary operator in $D$ and $U=\lim _{l \rightarrow \infty} U_{l}, U_{l} \in$ $D_{l} \simeq \bigotimes_{i=1}^{l} D_{i}$, where $D_{i}$ is the diagonal subalgebra of $M_{n_{i}}$. Then

(a)

(b)

$$
\left.\lambda_{U}\right|_{\mathbf{A}_{k}}=\operatorname{Ad}\left(U^{*} \Phi\left(U^{*}\right) \cdots \Phi^{k-1}\left(U^{*}\right)\right),
$$

$$
\lambda_{U}(x)=\lim _{l \rightarrow \infty} \operatorname{Ad}\left(U_{l}^{*} \Phi\left(U_{l}^{*}\right) \cdots \Phi^{k-1}\left(U_{l}^{*}\right)\right)(x)=\lim _{l \rightarrow \infty} \lambda_{U_{l}}(x) \text { for } x \in \mathbb{A}_{k} .
$$

Proof. (b) follows obviously from (a).

We prove (a) by induction of $k$. Because of $\lambda_{U}(x)=U^{*} x U$ for $x$ in $\mathbb{A}_{1}$, it is obvious that (a) holds for $k=1$. Suppose that (a) holds for $k-1$. We show (a) holds for $k$. Let an arbitrary element in $\mathbb{A}_{k}$ be the form $x \otimes y$, where 
$x$ is in $\mathbb{A}_{k-1}$ and $y$ is an $n \times n$ matrix, i.e., $y=\left(\beta_{i j}\right)$. Note that

$$
x \otimes y=\sum_{i, j=1}^{n} \beta_{i j} S_{i} x S_{j}^{*}
$$

and

$$
\begin{aligned}
\lambda_{U}(x \otimes y)=\sum_{i, j=1}^{n} \beta_{i j} U^{*} S_{i} \lambda_{U}(x) S_{j}^{*} U \\
=\sum_{i, j=1}^{n} \beta_{i j} U^{*} S_{i} A d\left(U^{*} \Phi\left(U^{*}\right) \cdots \Phi^{k-2}\left(U^{*}\right)\right)(x) S_{j}^{*} U \\
=U^{*}\left\{\sum_{i, j=1}^{n} \beta_{i j} S_{i}\left(U^{*} \Phi\left(U^{*}\right) \cdots \Phi^{k-2}\left(U^{*}\right)\right) x\left(\Phi^{k-2}(U) \cdots \Phi(U) U\right) S_{j}^{*}\right\} U \\
=U^{*}\left\{\sum_{i, j=1}^{n} \beta_{i j} \Phi\left(U^{*} \Phi\left(U^{*}\right) \cdots \Phi^{k-2}\left(U^{*}\right)\right) S_{i} x S_{j}^{*} \Phi\left(\Phi^{k-2}(U) \cdots \Phi(U)\right\} U\right. \\
=\sum_{i, j=1}^{n} \beta_{i j} A d\left(U^{*} \Phi\left(U^{*}\right) \cdots \Phi^{k-1}\left(U^{*}\right)\right)\left(S_{i} x S_{j}^{*}\right) \\
=\operatorname{Ad}\left(U^{*} \Phi\left(U^{*}\right) \cdots \Phi^{k-1}\left(U^{*}\right)\right)\left(\sum_{i, j=1}^{n} \beta_{i j} S_{i} x S_{j}^{*}\right) .
\end{aligned}
$$

Let $x$ be in $\mathbb{A}_{k}$ and $x=\left(\alpha_{i j}\right), i, j=1, \ldots, n^{k}$.

Then

$$
P(x)=\left[\begin{array}{cccc}
\alpha_{11} & 0 & \cdots & 0 \\
0 & \alpha_{22} & \cdots & 0 \\
\vdots & \vdots & \ddots & \vdots \\
0 & 0 & \cdots & \alpha_{n^{k} n^{k}}
\end{array}\right]=\operatorname{diag}(x) .
$$

3.2. Proposition. Let $\theta=\lambda_{U}$ for some unitary operator $U$ in $D$. Then $P \circ \theta=$ $P$.

Proof. Let $A$ be in $\mathbb{A}_{k}$ and $V$ be a unitary operator in $D_{k}$. We observe that

(1) $\lambda_{V}(A) \in \bigcup_{j=1}^{\infty} \mathbb{A}_{j}$.

(2) For $l>0, \lambda_{V}\left(\left(S_{1}^{*}\right)^{l} A\right)=\left(S_{1}^{*} V\right)^{l} \lambda_{V}(A) \notin \mathbb{A}=\bigcup_{j=1}^{\infty} \mathbb{A}_{j}$, due to Lemma 3.1.

For $x \in \mathbb{A}$ with

$$
x=\sum_{-N}^{-1} S^{i} A_{i}+A_{0}+\sum_{1}^{N} A_{i} S^{i}, \quad A_{i} \in \bigcup_{j=1}^{\infty} \mathbb{A}_{j},
$$

and

$$
U=\lim _{l \rightarrow \infty} U_{l}, \quad U_{l} \in D_{i},
$$


we have

$$
\begin{aligned}
P \circ \lambda_{U}(x) & =\lim _{l \rightarrow \infty} P \circ\left(\lambda_{U_{l}}(x)\right) \\
& =\lim _{l \rightarrow \infty} P \circ\left(\lambda_{U_{l}}\left(A_{0}\right)\right) \\
& =\lim _{l \rightarrow \infty} \operatorname{diag} A d\left(U_{l}^{*} \Phi\left(U_{l}^{*}\right) \cdots \Phi^{k-1}\left(U_{l}^{*}\right)\right)\left(A_{0}\right)
\end{aligned}
$$

Since $U_{l}^{*} \Phi\left(U_{l}^{*}\right), \ldots, \Phi^{k-1}\left(U_{l}^{*}\right)$ is a diagonal matrix in $A_{t}$ for some large $t \geq$ $k$, realizing $A_{0} \in \mathbb{A}_{t}$ we see that the diagonal entries in $A_{0}$ are fixed under $\operatorname{Ad}\left(U_{l}^{*} \Phi\left(U_{l}^{*}\right), \ldots, \Phi^{k-1}\left(U_{l}^{*}\right)\right)$. Hence

$$
P \circ \lambda_{U}(x)=\lim _{l \rightarrow \infty} \operatorname{diag}\left(A_{0}\right)=P(x) .
$$

Since $A$ is norm-dense in $O_{n}$, we have $P \circ \lambda_{U}=P$. Q.E.D.

3.3. Theorem. $\lambda_{U}$, for some unitary operator $U$ in $D$, is weakly inner on $O_{n}$.

Proof. Let $\Delta$ denote the pure-state space of $D$. Thus, $\Delta$ consists of all infinite sequences, $w=\left(j_{1}, j_{2}, \ldots\right)$, with $j_{i} \in\{1,2, \ldots, n\}$ for $i=1,2, \ldots$. For any $w \in \Delta$, we note that the state on $O_{n}$ defined by $w \circ P$ is $\lambda_{U}$-invariant for any unitary operator $U \in D$, for $(w \circ P) \circ \lambda_{U}=w \circ\left(P \circ \lambda_{U}\right)=w \circ P$ by Proposition 3.2. Hence, it suffices to show $w \circ P$ is a pure state on $O_{n}$ for some $w \in \Delta$ to establish that $\lambda_{U}$ is weakly inner on $O_{n}$ by Remark 2.4 . For the rest of the proof, we let $w=\left(j_{1}, j_{2}, \ldots\right)$ be a fixed element in $\Delta$ and $\phi=w \circ P$. We show that $\phi$ is pure.

Let the GNS representation triple induced by $\phi$ be denoted by $\{\pi, K, \xi\}$. We denote $\left(j_{1}, j_{2}, \ldots, j_{k}\right)$ by $\mu_{k}$ for $k=0,1, \ldots$. For $\nu \in W_{k}^{n}, \nu=$ $\left(i_{1}, \ldots, i_{k}\right)$, and $\eta \in W_{l}^{n}, \eta=\left(i_{1}, \ldots, i_{l}\right)$ with $l<k$, we let $\left(i_{l+1}, \ldots, i_{k}\right)$ be denoted by $\nu \backslash \eta$. We observe that the following two equivalence classes are the same: $\left[S_{\nu} S_{\mu_{l}}^{*} \xi\right],\left[S_{\eta} S_{\mu_{k}}^{*} \xi\right]$ if $\nu \backslash \eta=\mu_{l} \backslash \mu_{k}$ or $\eta \backslash \nu=\mu_{k} \backslash \mu_{l}$, and hence it is denoted by $(\nu, l)$. We also note that the following family of vectors in $K$ is orthonormal, $\{(\nu, l)\}$, for $\left\langle\pi\left(S_{\nu_{1}}\right) \pi\left(S_{\mu_{l}}^{*}\right) \xi, \pi\left(S_{\nu_{2}}\right) \pi\left(S_{\mu_{k}}^{*}\right) \xi\right\rangle=\phi\left(S_{\mu_{k}} S_{\nu_{2}}^{*} S_{\nu_{1}} S_{\mu_{l}}^{*}\right)=$ $\delta_{\left(\nu_{1}, l\right),\left(\nu_{2}, k\right)}$, for any $\nu_{1}, \nu_{2} \in W$. Since $\left\|\pi\left(S_{\eta} S_{\nu}^{*}\right) \xi\right\|=0$ for any $\eta, \nu \in W$ with $\nu \neq \mu_{k}, k=0,1, \ldots$, it follows that the orthonormal family mentioned above is actually an orthonormal basis for $K$. For any operator $T \in K$, we denote $T$ by $\left[T_{\nu l, \eta k}\right]$, where $T_{\nu l, \eta k}=\left\langle T \pi\left(S_{\nu} S_{\mu_{l}}^{*}\right) \xi, \pi\left(S_{\eta} S_{\mu_{k}}^{*}\right) \xi\right\rangle$. Let $T$ be an arbitrary operator in $\pi\left(O_{n}\right)^{\prime}$, and we may assume that $T(\xi)=\pi(x)(\xi)$ for some $x \in O_{n}$. Then $T_{\nu l, \nu l}=\left\langle T \pi\left(S_{\mu_{l}} S_{\mu_{l}}^{*}\right) \xi, \xi\right\rangle=\phi\left(S_{\mu_{l}} S_{\mu_{l}}^{*} x\right)=w\left(S_{\mu_{l}} S_{\mu_{l}}^{*} P(x)\right)=$ $w\left(S_{\mu_{l}} S_{\mu_{l}}^{*}\right) w(P(x))=w(P(x))=\langle T \xi, \xi\rangle$, denoted by $T_{0,0}$. Finally, we show $\left(T^{*} T\right)_{\nu l, \eta k}=0$ for all $T \in \pi\left(O_{n}\right)^{\prime}$, when $(\nu, l) \neq(\eta, k)$. Here we may assume that $T(\xi)=\pi(x)(\xi)$ for some $x \in O_{n}$. In fact, $\left(T^{*} T\right)_{\nu l, \eta k}=\left\langle\pi\left(S_{\nu} S_{\mu_{l}}^{*}\right) \pi(x) \xi\right.$, $\left.\pi\left(S_{\eta} S_{\mu_{k}}^{*}\right) \pi(x) \xi\right\rangle=\phi\left(x^{*} S_{\mu_{k}} S_{\eta}^{*} S_{\nu} S_{\mu_{l}}^{*} x\right)=0$, when $(\nu, l) \neq(\eta, k)$. Then, $T^{*} T$ is a scalar multiple of the identity operator for all $T \in \pi\left(O_{n}\right)^{\prime}$, and $\pi\left(O_{n}\right)^{\prime}$ consists of scalars of the identity operator. Therefore, $\pi$ is irreducible, and $\phi$ is pure. Q.E.D.

3.4. Remark. A different proof for some of the above $\phi$ 's that are pure can be seen in [12] (see Proposition 3.3 in [12]). A stronger result of pure-state extension of $w$ is also stated in [5] (see Proposition 3.1 in [5]). However, there is no proof provided for Proposition 3.1 in [5]. 
3.5. Corollary. Let $V$ be a unitary operator on $C^{n}$. Then $O_{V}$ is weakly inner. Proof. If $V$ is diagonal, then $O_{V}=\lambda_{V^{*}}$ and $O_{V}$ is weakly inner by Theorem 3.3, for $V \in D$ and $\varphi \circ P$ is a $O_{V}$-invariant pure state for any pure state $\varphi$ on $D$. In general, there is a unitary operator $W$ on $C^{n}$ such that $W V W^{*}$ is diagonal. After Remark 2.4 and an observation that $O_{W V W^{*}}=O_{W} O_{V} O_{W^{*}}=$

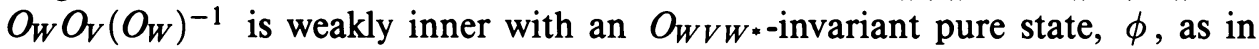
Theorem 3.3, we see that $O_{V}$, having an $O_{V}$-invariant pure state, $\phi \circ O_{W}$, is weakly inner. Q.E.D.

3.6. Remark. It follows from Remark 2.4 that any automorphism of $O_{n}$ conjugate to $\lambda_{U}$ in Theorem 3.3 is weakly inner. A result similar to Corollary 3.5 can also be found in [1], in which the approach is quite different.

\section{FOCK REPRESENTATION OF $O_{\infty}$}

For any unitary operator $V$ on $C^{n}, n=2,3,4, \ldots$, we can define an *-automorphism $O_{V}$ on $O_{\infty}$ by $O_{V}\left(S_{i}\right)=\sum_{k=1}^{n} V_{k i} S_{k}, i=1,2, \ldots, n$. Likewise, any permutation $V$ (may be infinite) of $\{2,3,4, \ldots\}$ induces an *automorphism $O_{V}$ in the obvious way by permuting the generators according to $V$. In this section we show that the $O_{V}$ of $O_{\infty}$ mentioned above is weakly inner. Let $H$ be an infinite-dimensional separable Hilbert space. Consider the FOCK space, $F(H)$, which is defined to be $\bigoplus_{r=0}^{\infty}\left[\otimes^{r} H\right]$, where $\otimes^{r} H$ is the Hilbert space tensor product of $r$ copies of $H$ and $\otimes^{0} H$ is a one-dimensional Hilbert space $C$ spanned by a unit vector $\Omega$, the vacuum. Define a linear map $O_{F}: H \rightarrow B(F(H))$ by

$$
\begin{aligned}
O_{F}(f)\left(f_{1} \otimes f_{2} \otimes \cdots \otimes f_{r}\right) & =f \otimes f_{1} \otimes \cdots \otimes f_{r}, \\
O_{F}(f)(\Omega) & =f .
\end{aligned}
$$

Let $\left\{h_{1}, h_{2}, \ldots\right\}$ be an orthonormal basis of $H$ and $P_{0}$ be the orthogonal projection of $H$ onto $\otimes^{0} H$. Then we have

$$
\begin{aligned}
& O_{F}(f)^{*} O_{F}(g)=\langle g, f\rangle I \quad \text { for all } f, g \in H, \\
& \sum_{i=1}^{\infty} O_{F}\left(h_{i}\right) O_{F}\left(h_{i}\right)^{*}+P_{0}=I
\end{aligned}
$$

where the infinite sum is taken in the strong-operator topology.

Comparing (4.1) with (2.2) we conclude that $O_{F}$ induces a faithful representation $\pi$ of $O_{\infty}$ by $\pi\left(S_{i}\right)=O_{F}\left(h_{i}\right), i=1,2, \ldots$, which is called the FOCK representation of $O_{\infty}$.

4.2. Lemma. (1) $\{\Omega\} \cup\left\{h_{i} \mid i=1,2, \ldots\right\} \cup\left\{h_{i} \otimes h_{j} \mid i, j=1,2, \ldots,\right\} \cup \ldots$ form an orthonormal basis for $F(H)$.

(2) $P_{0} \in \pi\left(O_{\infty}\right)^{\prime \prime}$.

(3) For any element $x$ in the above orthonormal basis there exists a rank-one partial isometry $T_{x}$ in $\pi\left(O_{\infty}\right)^{\prime \prime}$ such that $T_{x}$ maps $\otimes^{0} H$ onto the subspace spanned by $x$.

Proof. (1) is obvious.

(2) follows from the second condition in (4.1).

(3) Suppose $x=h_{i_{1}} \otimes h_{i_{2}} \otimes \cdots \otimes h_{i_{k}}$; then

$$
T_{x}=O_{F}\left(h_{i_{1}}\right) O_{F}\left(h_{i_{2}}\right) \cdots O_{F}\left(h_{i_{k}}\right) P_{0} \text {. Q.E.D. }
$$


4.3. Lemma. (1) $\pi\left(O_{\infty}\right)^{\prime \prime}=B(F(H))$.

(2) The vector state of $\pi\left(O_{\infty}\right), w_{\Omega}(A)=\langle A \Omega, \Omega\rangle$, is $\beta$-invariant for $\beta=$ $O_{V}$ where $V$ is a unitary operator in $C^{n}$ for some $n$ or a permutation of $\{1,2,3, \ldots\}$.

Proof. (1) follows from (3) in Lemma 4.2, for $\pi\left(O_{\infty}\right)^{\prime}=$ \{scalars $\left.\cdot I\right\}$.

(2) Any word consisting of $\pi\left(S_{i}\right), \pi\left(S_{i}\right)^{*}, i=1,2, \ldots$, can be reduced to $\alpha I$ for some scalar $\alpha$ or $\pi\left(S_{j_{1}}\right) \cdots \pi\left(S_{j_{l}}\right) \pi\left(S_{i_{1}}\right)^{*} \cdots \pi\left(S_{i_{m}}\right)^{*}$ with $l \geq 1$ or $m \geq$ 1. The null space, $N$, of the bounded linear functional $w_{\Omega}$ is a closed subspace spanned by words in their reduced form $\pi\left(S_{j_{1}}\right) \cdots \pi\left(S_{j_{l}}\right) \pi\left(S_{i_{1}}\right)^{*} \cdots \pi\left(S_{i_{m}}\right)^{*}$ with $l \geq 1$ or $m \geq 1$. It is easy to see that $N$ is invariant under $\beta$ described in (2) of Lemma 4.3. Thus $w_{\Omega}$ and $w_{\Omega} \circ \beta$ have the same null space and $w_{\Omega}(I)=w_{\Omega} \circ \beta(I)=1$. Hence $w_{\Omega}=w_{\Omega} \circ \beta$. Q.E.D.

4.4. Theorem. Every automorphism $O_{V}$ of $O_{\infty}$, with $V$ being a unitary operator on $C^{n}$ for some $n$ or a permutation of $\{1,2,3, \ldots\}$, is weakly inner.

Proof. From (1), (2) of Lemma 4.3 we see that $\pi$ is an irreducible representation of $O_{\infty}$ and $w_{\Omega} \circ \pi$ is an $O_{V}$-invariant pure state of $O_{\infty}$. Hence by Remark 2.4, Theorem 4.4 is proved. Q.E.D.

4.5. Concluding remark. The similar construction of FOCK representation for $O_{n}, n$ : finite, does not yield the same result. The FOCK representation for $O_{\infty}$ can also be found in [8]. A result like Theorem 4.4 is also recorded in [1] with a different proof. It also follows from Remark 2.4 that any automorphism of $O_{\infty}$ conjugate to $O_{V}$ in Theorem 4.4 is weakly inner.

\section{REFERENCES}

1. H. Araki, A. L. Carey, and D. Evans, On $O_{n+1}$, J. Operator Theory 12 (1984), 243-264.

2. R. F. Archbold, On the "flip-flop" automorphisms of $C^{*}\left(S_{1}, S_{2}\right)$, Quart. J. Math. Oxford Ser. (2) 30 (1979), 129-132.

3. Joachim Cuntz, Simple $C^{*}$-algebras generated by isometries, Comm. Math. Phys. 57 (1977), 173-185.

4. __ Murray-von Neumann equivalence of Projections in infinite simple $C^{*}$-algebras, Rev. Roumaine Math. Pures Appl. 23 (1978), 1011-1014.

5. —_, Automorphisms of certain simple $C^{*}$-algebras, Quantum Fields-Algebras-Processes (L. Streit, ed.), Springer-Verlag, Berlin and New York.

6. M. Enomoto, H. Takehana, and Y. Watatani, Automorphisms on Cuntz algebras, Math. Japon. 24 (1979), 231-234.

7. M. Enomoto, M. Fujii, H. Takehana, and Y. Watatani, Automorphisms on Cuntz algebras. II, Math. Japon. 24 (1979), 463-468.

8. D. Evans, On $O_{n}$, Publ. Res. Inst. Math. Sci. 16 (1980), 915-927.

9. R. Kadison and J. Ringrose, Derivations and automorphisms of operator algebras, Comm. Math. Phys. 4 (1967), 32-63.

10. A. Kishimoto, Outer automorphisms and reduced crossed products of simple $C^{*}$-algebras, Comm. Math. Phys. 81 (1981), 429-435.

11. E. C. Lance, Inner automorphisms of UHF algebras, J. London Math. Soc. 43 (1968), 681-688.

12. A. Lazar, S.-K. Tsui, and S. Wright, Pure state extensions and restrictions in $O_{2}$, J. Operator Theory 23 (1990), 171-178.

Department of Mathematical Sciences, Oakland University, Rochester, Michigan 48309-4401

E-mail address: tsui@vela.acs.oakland.edu 